\title{
Systemic Hormonal Unloading in Unilateral Adrenalectomy in a Patient with Bilateral Adrenal Hyperplasia: A Case Report

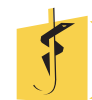

\author{
Ma. Felisse Carmen Gomez, M.D. ', \\ Florence Rochelle Gan, M.D. ${ }^{2}$, \\ Erick Mendoza, M.D. ${ }^{3}$, \\ Leilani B. Mercado Asis, M.D., Ph.D., MPH ${ }^{4}$
}

\begin{abstract}
Background Unilateral adrenalectomy has not been recommended in the guidelines as a treatment for primary hyperaldosteronism secondary to bilateral adrenal hyperplasia (BAH). Interestingly, recent studies have shown that increased circulation of aldosterone increased oxidative stress, cardiovascular (CV) complications such as atrial fibrillation, myocardial infarction and heart failure; and that unilateral adrenalectomy led to improved CV function. Therefore, recognizing the role of unilateral adrenalectomy in $\mathrm{BAH}$, specifically for improved quality of life is important.
\end{abstract}

Clinical case A 47-year-old hypertensive (highest blood pressure [BP] 150/90 $\mathrm{mmHg}$ ) woman had a severe headache, muscle weakness, polyuria, and

$\triangle$ Ma. Felisse Carmen S. Gomez

felitchi5@yahoo.com

1 University of Santo Tomas Hospital, Internal Medicine -

Section of Endocrinology and Metabolism

2 University of Santo Tomas Hospital, Section of Endocrinology, Diabetes and Metabolism, Department of Internal Medicine

3 Department of Internal Medicine and Endocrinology, University of Santo Tomas

4 Faculty of Medicine and Surgery, University of Santo Tomas polydipsia. Her serum potassium (K) was low at 3.1 $\mathrm{mmol} / \mathrm{L}$ (3.5-5 mmol/L). Initial tests showed elevated plasma aldosterone, suppressed plasma renin activity and elevated aldosterone-renin ratio $(6.61 \mathrm{ng} /$ $\mathrm{dL},<0.1 \mathrm{ng} / \mathrm{mL}$ and 66 , respectively). Plasma aldosterone after saline suppression test $(12.70 \mathrm{ng} / \mathrm{dL})$ confirmed the diagnosis of primary aldosteronism (PA). MRI showed a well-defined, oval-shaped solid nodule in the medial limb of the left adrenal gland $(1.8 \times 1.2 \mathrm{~cm})$. Bilateral adrenal vein sampling with adrenocorticotropic hormone (ACTH) stimulation test was compatible with BAH (cortisol-corrected aldosterone ratio pre-ACTH stimulation 1.29 and postACTH 1.66), with dominant aldosterone secreting left adrenal gland (7200 vs $3760 \mathrm{ng} / \mathrm{dL}$ ). She was started on spironolactone $200 \mathrm{mg} /$ day and amlodipine $10 \mathrm{mg} /$ day and eventually shifted to eplerenone. Despite the optimal dose of eplerenone and amlodipine, she still experienced severe headaches, palpitations and breakthrough elevations of BP that led to her recurrent admissions. Eplerenone was shifted back to spironolactone (150-200 mg/day) with amlodipine dose (10 mg/day) normalizing her blood pressure and potassium level, yet with persistent headache and muscle weakness. Repeat imaging using $C T$ scan with contrast showed consistent results. Postoperatively, with all medications discontinued the patient was asymptomatic, normotensive 
$(110 / 70 \mathrm{mmHg})$ and normokalemic $(4.0 \mathrm{mmol} / \mathrm{L})$. One month later, her BP started to increase again at $140 / 80 \mathrm{mmHg}$ and her $\mathrm{K}$ decreased to $3.4 \mathrm{mmol} / \mathrm{L}$. Normalization of said parameters (BP:120/70 $\mathrm{mmHg} \mathrm{K:} 4.1 \mathrm{mmol} / \mathrm{L}$ ), with stabilization following lower doses of amlodipine (5mg/day) and spironolactone (25 mg/day). Also, all the symptomatology of the patient resolved completely.

Conclusion This present case exemplifies a unilateral adrenalectomy approach in $\mathrm{BAH}$, which led to improvement in BP and $\mathrm{K}$ levels, despite low medication doses. Furthermore, symptom relief and improved quality of life, as desired outcomes, were achieved.

Keywords Hormonal unloading, primary aldosteronism, hypertension, bilateral adrenal hyperplasia, hyperaldosteronism, case report

\section{INTRODUCTION}

Hypertension is a serious public health problem that affects more than 1 billion patients worldwide.[1] Arterial hypertension of unknown origin or commonly known as primary or "essential" hypertension is seen in a majority of cases, about $85 \%$, and is considered secondary in a small fraction of hypertensive patients, approximately 15\%.[2]

The secondary causes of hypertension can be divided into renal causes, such as renal parenchymal or renovascular disease, and endocrine causes. There are at least 14 endocrine disorders for which hypertension may be the initial clinical presentation. [2] Primary aldosteronism (PA) is a common form of endocrine hypertension in which aldosterone production is inappropriate and at least partially autonomous of the renin-angiotensin system. PA is the most common cause of secondary hypertension and approximately $5-12 \%$ of all people with high BP have it. $[3,4,5]$ A proper diagnosis of PA is very important for clinicians because it provides an opportunity to either cure hypertension or uses targeted pharmacotherapy and prevents complications such as renal failure and cardiac disease.[2,3] Aldosterone-producing adenoma (APA) and bilateral idiopathic hyperaldosteronism $(\mathrm{IHA})$ are the most common subtypes of primary aldosteronism, approximately $30 \%$ and $60 \%$, respectively. [2] APA is treated surgically and IHA is mainly managed medically. [2] This present case report is hinged on the fact that there is no local data and there is paucity of reported cases worldwide regarding $\mathrm{BAH}$. This present case also exemplifies BAH being non-responsive to aldosterone receptor antagonist, yet surgically manageable through hormonal unloading.

Informed consent was taken voluntarily and done in accordance with the Data Privacy Act of 2012.

\section{CLINICAL CASE}

A 47-year-old woman was admitted due to elevated BP $150 / 90 \mathrm{mmHg}$. Nine years ago, she was diagnosed with uncontrolled blood pressure and started on three antihypertensive medications including amlodipine $10 \mathrm{mg} / \mathrm{tab} 1 \mathrm{tab} O D$, losartan 50 $\mathrm{mg} / \mathrm{tab} 1 \mathrm{tab} O D$, hydrochlorothiazide $12.5 \mathrm{mg} /$ tab 1 tab BID. She also had mild hypokalemia (K: $3.1 \mathrm{mmol} / \mathrm{L}$, N.V. 3.7-5.0). No chest pain, no dyspnea, no congestive symptoms, no palpitations, no headache, and no sweating were noted; however, she had polyuria and polydipsia. Three years prior to admission, an MRI of the whole abdomen was done, which showed a well-defined oval-shaped solid nodule in the medial limb of the left adrenal $1.8 \times 1.2 \mathrm{~cm}$; right side notably normal. The patient was eventually referred to an endocrinologist who did the following work ups: urine metanephrines: negative, serum cortisol $1.2 \mathrm{mcg} / \mathrm{dL}$ after $1 \mathrm{mg}$ dexamethasone suppression, plasma aldosterone 6.61 $\mathrm{ng} / \mathrm{dL}$, renin $<0.1 \mathrm{ng} / \mathrm{mL}, A R R$ 66. The patient was assessed to have primary aldosteronism (PA) and a confirmatory test was done. The saline suppression test result was elevated at 12.70, which was compatible with the diagnosis. The patient was started on spironolactone $50 \mathrm{mg} / \mathrm{tab} 1 \mathrm{tab}$ OD. At this time, she was advised surgery but opted to seek a second opinion with another endocrinologist. Two years prior to admission, bilateral adrenal vein sampling with ACTH stimulation test was done and the result showed BAH (cortisol-corrected aldosterone ratio pre-ACTH stimulation 1.29 and post ACTH 1.66). She was managed medically with spironolactone $100 \mathrm{mg} / \mathrm{tab} 1$ tablet twice daily, but developed gastrointestinal upset and amenorrhea. She consulted another endocrinologist and spironolactone was shifted to eplerenone $50 \mathrm{mg} /$ tab 1 tablet twice daily. Three months prior to admission, the patient had a severe headache and high BP with palpitations. She was admitted and repeat urine metanephrines done which still showed a negative result. Eplerenone was 


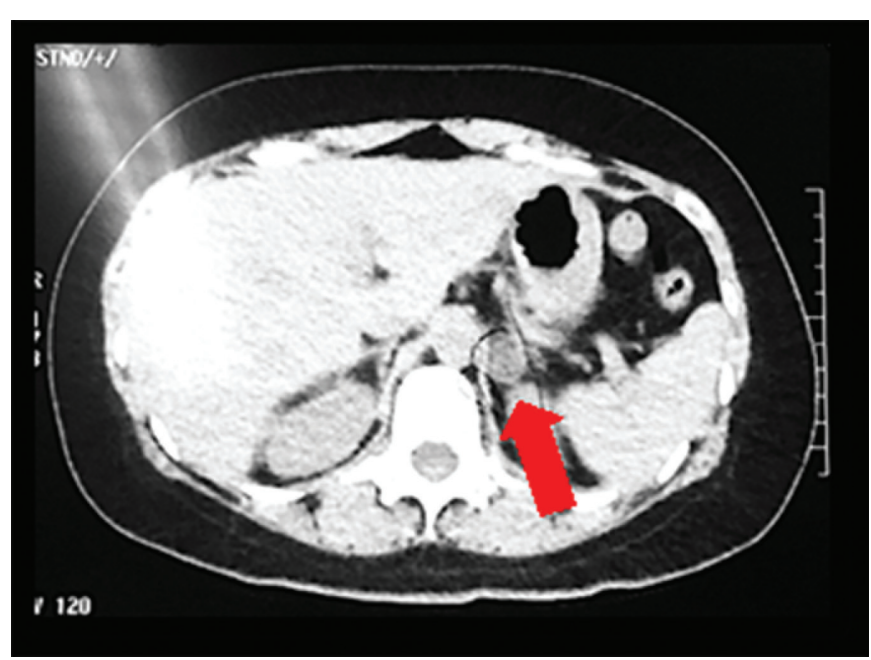

Figure 1. CT scan of the whole abdomen with contrast (Red arrow points to the $1.5 \times 2.2 \mathrm{~cm}$ nodule on the left adrenal gland)

shifted back to spironolactone $100 \mathrm{mg} /$ tab 1 tablet in the morning and $50 \mathrm{mg} / \mathrm{tab} 1$ tablet in the afternoon. Computed tomography scan of the upper abdomen with contrast showed a $1.5 \times 2.2 \mathrm{~cm}$ nodule on the left adrenal gland on pre-contrast with mild enhancement at $60 \mathrm{sec}$ and rapid washout at 15 min delayed study. This is consistent with adrenal adenoma (Figure 1). The patient was advised to undergo unilateral adrenalectomy; hence admission.

On physical examination, the patient was conscious, coherent, and comfortable. BP was 140/80 $\mathrm{mmHg}$, heart rate $96 \mathrm{bpm} /$ regular, respiratory rate of 16 and afebrile. The patient had a height of 1.62 $\mathrm{m}$, weighed $63.5 \mathrm{~kg}$ with a BMl of $24 \mathrm{~kg} / \mathrm{m}^{2}$.

She had warm, moist skin, no moon facies, no facial plethora, pink palpebral conjunctivae, anicteric sclerae, no periorbital edema; no exophthalmos, no lid lag, and no retraction. Her pupils were equally reactive to light. There was no hirsutism. She had non-distended neck veins, symmetrical chest expansion, no galactorrhea, no breast mass nor nipple discharge, no retractions, and no crackles or wheezes. She had adynamic precordium; apex beat was at the 5th LICS MCL, no heaves, lifts or thrills, with loud S1 followed by soft S2 at the apex, loud S2 followed by soft S1 at the base. No cardiac murmurs appreciated. Her abdomen was protuberant, flabby with whitish abdominal striae. She had a soft and non-tender abdomen; the liver span was $8 \mathrm{~cm}$, right MCL; Traube's space was obliterated. There were no hand tremors, no joint swelling. The pulses were full and equal with normal deep tendon reflexes ++ on all extremities.

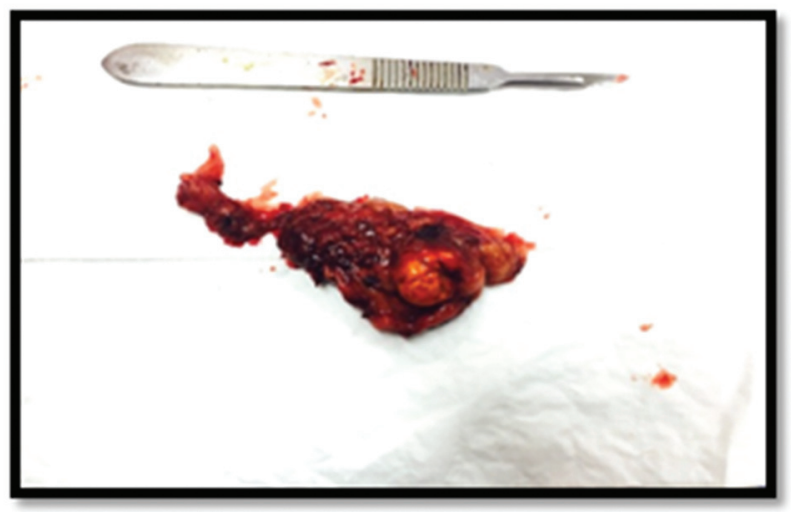

Figure 2. Adrenal Gland

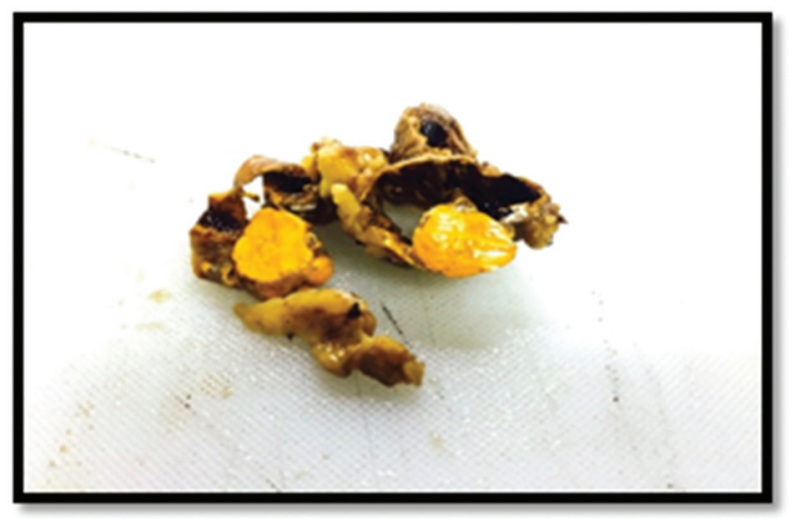

Figure 3. Cross section cut of the adrenal gland, left (well circumscribed, yellow tumor in the adrenal cortex)

The patient underwent laparoscopic left adrenalectomy on the second hospital day. Her operation was unremarkable. Intraoperative findings: The left adrenal gland measured $7 \times 1.5 \times 2 \mathrm{~cm}$ with a palpable mass $1.5 \times 1.5 \mathrm{~cm}$ noted on the posteromedial aspect (Figure 1 and 2 ).

Postoperatively, the serum $K$ was normal at 3.9 $\mathrm{mmol} / \mathrm{L}$. The patient was asymptomatic for the rest of the hospital stay. Her BP ranged from 100-120/60$80 \mathrm{mmHg}$. The patient was discharged on the third hospital day without the need for spironolactone or antihypertensive medications. The left adrenal gland histopathology showed loose clusters of cells with round to oval, small, smooth, benign-appearing nuclei and abundant foamy, vacuolated cytoplasm compatible with adrenal adenoma (Figure 4).

Postoperatively, the patient was normotensive (BP $110 / 70 \mathrm{mmHg}$ ) and normokalemic (4.0 mmol/L). On follow up after one month without taking any antihypertensive medications and spironolactone, she still maintained BP and normal 


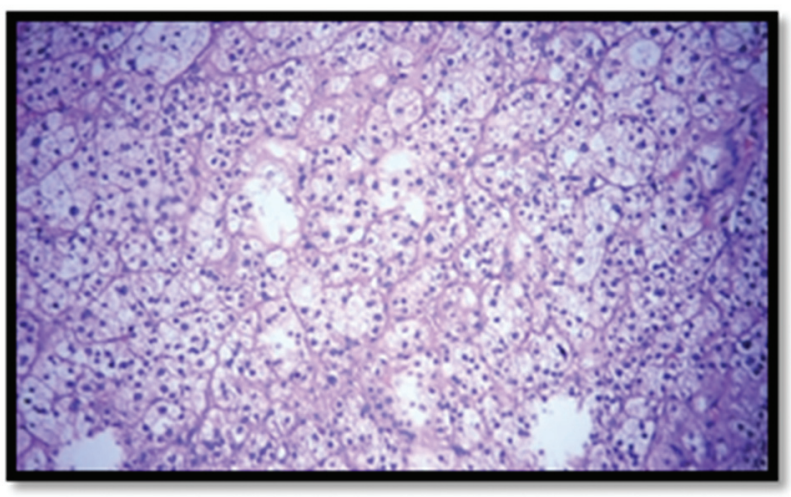

Figure 4. Histopathology of the left adrenal gland (distinct cell borders with large cells having different cytoplasm content and with a variation of nuclear size)

$\mathrm{K}$ level (3.8 mmol/L). On follow up after two months, the patient had episodes of dizziness, BP of 160/90 and $\mathrm{K} 4.1 \mathrm{mmol} / \mathrm{L}$, spironolactone $25 \mathrm{mg}$ tab and amlodipine $5 \mathrm{mg}$ tab once daily was resumed. There was no recurrence of symptoms and her BP eventually normalized with a lower dose of spironolactone and antihypertensive medication.

\section{DISCUSSION}

Primary aldosteronism is the most frequent endocrine cause of secondary hypertension affecting almost $10 \%$ of all patients referred to specialized clinics. [6] In the Philippines, the prevalence of hypertension was $22.3 \%$ in 2013; [7] however, there is no data on the prevalence of secondary hypertension in the country, but in Japan, there was $9.1 \%$ prevalence of secondary hypertension as of 2004. [8]

In the study of Doroin, [9] they found that the clinical profile of patients with PA in a tertiary hospital showed weakness of extremities (89\%), headache $(11 \%)$, and the median baseline duration of hypertension was 48 months.

Autonomous aldosterone secretion by the adrenal glands results in hypokalemia, sodium reabsorption, and fluid retention. The alarming forthcoming trouble in this situation is that prolonged exposure to high aldosterone concentrations has a deleterious effect on cardiovascular tissues and is associated with target organ damage. $[10,11,12]$

The study of Savard [11] found that the frequency of CV events was significantly higher in patients with PA than in properly matched controls with essential hypertension. A history of atrial fibrillation, myocardial infarction, coronary artery disease, or heart failure was more frequently found at the first visit in patients with PA. The culprit to this is probably secondary to the stress, endothelial dysfunction, inflammation, remodeling, hypertrophy, and fibrosis brought about by the high secretion of aldosterone. $[12,13]$ These mechanisms lead to structural abnormalities, including an increase in arterial wall stiffness and carotid intima media thickness, the formation of carotid plaques, and myocardial fibrosis. $[13,16]$

For $\mathrm{BAH}$, it has been emphasized that the treatment of choice is medical management; however, treatment with spironolactone has some adverse effects such as gynecomastia in males and menstrual irregularity in females. Moreover, BP control may not be completely achieved with spironolactone or eplerenone; [17] therefore, unilateral adrenalectomy is a treatment option, such as in the case of our patient.

This surgical option offered to a select number of patients is called systemic unloading. It refers to a process of reducing the load of hormonal secretion from an autonomously functioning gland. In cases of IAH who failed medical therapy, unilateral adrenalectomy aims to reduce the production of aldosterone and subsequently, the effect of aldosterone excess by the autonomously functioning adrenal gland.

The study of Sukor et al.[17] in 2009 examined the role of unilateral adrenalectomy in patients with bilateral PA in a single center over 22 years. Among the 40 patients included, the most common rationale for surgery was failed medical therapy, in the form of intolerant adverse effects of mineralocorticoid antagonists or uncontrolled hypertension in spite of maximizing antihypertensive medication. The adrenal chosen for removal was on either a finding of higher adrenal venous aldosterone/cortisol ratio on AVS or an adrenal nodule on CT scan of the abdomen, such as in our patient. On follow up, these patients were normokalemic and normotensive requiring less antihypertensive medication.[17]

The strength of this study lies in the good judgement of the medical team to offer surgery as a treatment option. The limitation of this study is the short followup on the case at the time of writing this case. Due to the underdiagnosed prevalence of secondary hypertension, only this case has been reported in the country.

In conclusion, hormonal unloading through removal of the dominant adrenal in BAH leads to im- 
proved quality of life and better control of BP on a lower dose of medication, as exemplified in this case report.

Patient Anonymity, Consent, and Confidentiality

Written informed consent was obtained from the patient voluntarily for writing and publication of this case report and the accompanying images (CT scan images and histopathology slide images). All the information regarding the patient was kept in strict confidence and patient identifiers (such as name, geographical location, date of birth, contact number, etc.) The patient's anonymity and confidentiality is protected by non-disclosure of any personal information that will identify the individual when the study is published or presented. A breach of confidentiality may occur if the information is used in any other way. 


\section{REFERENCES}

1. Athyros V, Doumas M, Karagiannis A, Papademetriou V. Arterial Stiffness and Emerging Biomarkers. Angiology. 2015;66(10):901-3.

2. Kronenberg H, Larsen PR, Melmed S, Polonsky K. Williams Textbook of Endocrinology. Philadelphia, PA: Elsevier; 2015.

3. Fardella CE, Gordon RD, Loh KC, Mulatero P, Stowasser M, et al. Increased Diagnosis of Primary Aldosteronism, Including Surgically Correctable Forms, in Centers from Five Continents [Internet]. OUP Academic. Oxford University Press; 2004.

4. Keh-Chuan, Kham, Koay E, Min-Cheh, Shantac E. Prevalence of Primary Aldosteronism among Asian Hypertensive Patients in Singapore 1 [Internet]. OUP Academic. Oxford University Press; 2000.

5. Fogari R, Preti P, Zoppi A, Rinaldi A, Fogari E, Mugellini A. Prevalence of primary aldosteronism among unselected hypertensive patients: a prospective study based on the use of an aldosterone/renin ratio above 25 as a screening test. [Internet]. Current neurology and neuroscience reports. U.S. National Library of Medicine; 2007 [cited 2018 Dec 28]. Available from: https://www.ncbi.nlm.nih.gov/ pubmed/17460380

6. Hannemann A, Wallaschofski H. Prevalence of primary aldosteronism in patient's cohorts and in population-based studies-a review of the current literature. [Internet]. Current neurology and neuroscience reports. U.S. National Library of Medicine; 2012 [cited 2018 Dec 28]. Available from: https://www.ncbi.nlm.nih.gov/pubmed/22135219

7. Gumaro M. 8th National Nutrition Survey [Internet]. FNRI Website. [cited 2018 Dec 28]. Available from: http:// www.fnri.dost.gov.ph/index.php/nutrition-statistic/19-nutrition-statistic/1 18-8th-national-nutrition-survey

8. Omura M, Saito J, Yamaguchi K, Kakuta Y, Nishikawa T. Prospective Study on the Prevalence of Secondary Hypertension among Hypertensive Patients Visiting a General Outpatient Clinic in Japan. Hypertension Research. 2004;27(3): 193-202.

9. Duroin P, Gomez HS, Mercado-Asis LB. Clinical Profile of Patients with Hyperaldosteronism at Santo Tomas University Hospital. Philippine Journal of Internal Medicine. 2002.
10. Rossi G, Boscaro M, Ronconi V, Funder JW. Aldosterone as a cardiovascular risk factor. Trends in Endocrinology \& Metabolism. 2005;16(3):104-7.Catena et al., Aldosterone and the heart: from basic research to clinical evidence. Horm Metab Res. 2012;44:181-187.

11. Milliez P, Giererd X, Plouin P-F, Blacher J, Safar M, Mourad J-J. Evidence for an Increased Rate of Cardiovascular Events in Patients With Primary Aldosteronism. ACC Current Journal Review. 2005;14(8):18. Savard et al., Cardiovascular Complications Associated With Primary Aldosteronism A Controlled Cross-Sectional Study. Hypertension. 2013;62:331-336.

12. Matsumura K, Fujii K, Oniki H, Oka M, lida M. Role of Aldosterone in Left Ventricular Hypertrophy in Hypertension. American Journal of Hypertension. 2006;19(1):13-8. Marney AM and Brown NJ. Aldosterone and end-organ damage. Clin Sci (Lond). 2007; 1 13:267-278.

13. Strauch B, Petrak O, Wichterle D, Zelinka T, Holaj R, Widimskyjr J. Increased Arterial Wall Stiffness in Primary Aldosteronism in Comparison With Essential Hypertension. American Journal of Hypertension. 2006; 19(9):909-14.

14. Sukor N, Gordon RD, Ku YK, Jones M, Stowasser M. Role of Unilateral Adrenalectomy in Bilateral Primary Aldosteronism: A 22-Year Single Center Experience. The Journal of Clinical Endocrinology \& Metabolism. 2009;94(7):2437-45.

(i) Open Access This article is licensed under a Creative Commons Attribution 4.0 International License, which permits use, sharing, adaptation, distribution and reproduction in any medium or format, as long as you give appropriate credit to the original author(s) and the source, provide a link to the Creative Commons license, and indicate if changes were made. The images or other third party material in this article are included in the article's Creative Commons license, unless indicated otherwise in a credit line to the material. If material is not included in the article's Creative Commons license and your intended use is not permitted by statutory regulation or exceeds the permitted use, you will need to obtain permission directly from the copyright holder. To view a copy of this license, visit http://creativecommons.org/licenses/ by/4.0/. 